\title{
Prevalence of Sleep-Related Disordered Breathing among Acromegaly Patients and its Correlation to Cephalometric Parameters: An Indian Perspective
}

\author{
Sreejith $\mathrm{M}^{1} \quad$ Mohd Ashraf Ganie ${ }^{1} \quad$ Ravinder Goswami \\ Devasenathipathy Kandasamy ${ }^{3}$
${ }^{1}$ Department of Endocrinology and Metabolism, All India Institute of Medical Sciences, New Delhi, India
2Department of Pulmonary Medicine and Sleep Disorders, All India Institute of Medical Sciences, New Delhi, India
${ }^{3}$ Department of Radiodiagnosis, All India Institute of Medical
Sciences, New Delhi, India

Ann Natl Acad Med Sci (India) 2020;56:9-14
Nikhil Tandon ${ }^{1}$ Randeep Guleria ${ }^{2}$

\begin{abstract}
Address for correspondence Mohd Ashraf Ganie, MBBS, MD, DM, FRCP, Department of Endocrinology and Metabolism, All India Institute of Medical Sciences, Ansari Nagar, New Delhi 110029, India (e-mail: ashraf.endo@gmail.com).
\end{abstract}

\begin{abstract}
Keywords

- sleep-related breathing disorder

- cephalometry

- acromegaly

- obstructive sleep apnea

- central sleep apnea

- Apnea-Hypopnea Index
\end{abstract}

Introduction Sleep-related breathing disorders (SRBDs) including obstructive sleep apnea (OSA) and central sleep apnea (CSA) are quite common and are the leading causes of mortality in acromegaly. OSA in acromegaly is generally attributed to changes in oropharyngeal soft tissues. Data on OSA in Indian acromegaly are scant, especially cephalometric findings. The aim of this study is to evaluate the burden of SRBDs in acromegaly and its correlation to cephalometric parameters.

Materials and Methods A total of 32 subjects ( 20 men and 12 women), diagnosed with acromegaly on the basis of standard clinical, biochemical, and hormonal measurements were recruited. In addition to the above parameters, polysomnography and magnetic resonance imaging (MRI) of the pharynx were performed in all subjects.

Results The mean age of the subjects was $42.66 \pm 11.13$ years (range $=26-66$ ) and mean duration of study after first presentation was $7.6 \pm 6.3$ years (range $=0.25-32$ ). A total of 28 of $32(93.3 \%)$ subjects had sellar MRI documented macroadenomas while $20(62.5 \%)$ patients were treatment naive at the time of assessment. Twenty-nine (90.6\%) patients had evidence of SRBD and all of them had OSA subtype. The Apnea-Hypopnea Index (AHI) indicating severity of OSA (mild 21.8\%, moderate $34.4 \%$, and severe $34.4 \%$ ) correlated positively with tongue length, uvula length, and uvula thickness on MRI. However, AHI had no correlation with the severity of $\mathrm{GH}$ excess or disease activity or individual parameters such as weight, body mass index, blood pressure, hemoglobin A1c, serum human growth hormone, and insulin-like growth factor- 1 level.

Conclusion SRBD, the generally overlooked comorbidity, is highly prevalent in subjects with acromegaly and is almost always due to OSA, the severity of which correlates positively with tongue and uvula size. Well-designed, long-term follow-up study on a large cohort of acromegalic patients is required to improve our understanding on the subject.
DOI https://doi.org/

10.1055/s-0040-1709606 ISSN 0379-038X.
(C2020 National Academy of Medical Sciences (India)
License terms

(요 $\odot \circledast$ 


\section{Introduction}

Acromegaly is a rare endocrine disorder characterized by unregulated secretion of growth hormone $(\mathrm{GH})$ leading to varied clinical manifestations. ${ }^{1}$ Overall mortality is increased up to threefold, with cardiovascular and respiratory disorders being the leading causes. ${ }^{2}$ Obstructive sleep apnea (OSA) and central sleep apnea (CSA) are typical sleep-related breathing disorders (SRBD) complicating acromegaly. ${ }^{3-7}$ OSA is present in up to $90 \%$ of patients with active acromegaly and is attributed to changes in soft, cartilaginous, and bony tissues at the level of craniofacial, pharyngeal, and laryngeal structures leading to obstruction of the airflow. ${ }^{8-12}$ In addition to the adenoid, uvular, and tonsillar hypertrophy, macroglossia also narrows pharyngeal airway space in acromegaly ${ }^{8-12}$ causing air inflow impediment. Data on acromegaly from India are scanty with a single study on a cohort of 271 acromegalic subjects reporting prevalence a of $10.3 \%$, based on clinical questionnaire, with no comment on SRBD. ${ }^{13}$ Therefore, in view of nonexistent data on SRBD in acromegaly patients from India, we attempted to study this subject utilizing polysomnography or imaging modalities.

\section{Materials and Methods}

This was a cross-sectional study enrolling consecutive patients of acromegaly attending the Department of Endocrinology and Metabolism, All India Institute of Medical Sciences, New Delhi from January 2016 to August 2017. The subjects presenting or referred with suspected symptoms or signs of acromegaly were informed about the study. Those volunteering to participate were required to sign an informed consent before enrolling in the study. The study was performed according to the Helsinki declaration of 1975 and the Institutional Ethics Committee duly approved the protocol.

\section{Subject Selection}

The adult (18-70 years) subjects with known acromegaly (treated or untreated) or those who came with suspicion and qualified a diagnosis of acromegaly on the basis of standard biochemical and imaging criteria as per the department protocol were included in the study.

\section{Clinical Assessment}

Subjects were evaluated in detail using a predesigned questionnaire recording details of medical facts such as history of onset of acral enlargement, headache, visual problems, hyperhidrosis, etc. in addition to demographic data such as age, sex, family history, drug intake, presence of known comorbidities like diabetes mellitus, hypertension, cardiovascular disease, etc. Besides recording of symptoms suggestive of SRBD like snoring, excessive day time sleepiness, etc. subjects were asked to fill STOP-Bang screening questionnaire. Patients who refused consent, had any known lung or CNS disease, antidepressant or sedative drug intake, untreated hypothyroidism, had critical illness were excluded from the study.

Physical examination included anthropometric parameters like height, weight, neck, waist and hip circumferences, measurement of BP, and a brief systemic examination emphasizing on clinical features of acromegaly (bony and soft tissue enlargement such as prognathism, acral enlargement, acrochordons, acanthosis, field of vision, etc.) and its systemic complications. Height was measured by stadiometer and weight by electronic weighing machine (SECA 813, Germany), measurement of blood pressure (Omron 714, Kyoto Japan). Waist circumference was measured midway between lowest margin of last rib and tip of iliac crest. Hip circumference was measured at the widest portion of the buttock with the tape parallel to the floor. Neck circumference was measured just below the laryngeal prominence perpendicular to the long axis of the neck..$^{14}$

\section{Laboratory Evaluation}

All the enrolled subjects had estimation of hemogram, HbA1c, electrolytes, glucose, lipids, liver, and renal function tests, in addition to post-oral glucose tolerance test human growth hormone (hGH) suppression test. Oral glucose tolerance test was done between 800 to 900 hours after an overnight fast by administering $75 \mathrm{gm}$ of anhydrous glucose, followed by $10 \mathrm{~mL}$ venous blood draw at 0,60 , and 120 minutes after glucose challenge. The samples were cold centrifuged, aliquoted, and immediately analyzed (blood counts and biochemistry) or stored at $-80^{\circ} \mathrm{C}$ until the assay (for serum $\mathrm{T} 4$, thyroid-stimulating hormone, prolactin, luteinizing hormone, follicle-stimulating hormone, total testosterone, estradiol, cortisol, human growth hormone [hGH], and insulin-like growth factor-1 [IGF-1]).

\section{Assays}

Fasting plasma glucose was estimated by hexokinase method using COBAS INTEGRA 400 plus analyzer (Roche Diagnostics, Indiana, United States), and HbA1c was done by high performance liquid chromatography (TOSOH G8 HPLC analyzer, Tosoh Bioscience Inc, San Francisco, California, United States). Liver function test, kidney function test, and other biochemical 
parameters were also analyzed on COBAS INTEGRA 400 plus analyzer using standard methodologies. Serum hGH, IGF-1, and other hormonal parameters (T4, thyroid-stimulating hormone, prolactin, luteinizing hormone, follicle-stimulating hormone, total testosterone, estradiol, and cortisol) were measured by electrochemiluminescent immunometric assay (CobasE 411 analyzer, Roche Diagnostics, Indiana, United States) using supplier protocol. Intraassay or interassay CVs were within the prescribed limits.

\section{Polysomnography}

Complete overnight polysomnography was done using ALICE 5 sleep system (San Diego, California, United States). Recordings included patient position, electroencephalography, and electrooculography for sleep staging, chin electromyography, nasal airflow by a sensor, chest and abdominal strain gauges for respiratory movements, electrocardiography and pulse oxymetry for blood oxyhemoglobin saturation. From the analysis of above recordings, the laboratory technician reported the polysomnogram by the Apnea-Hypopnea Index (AHI) score and was counterchecked by the pulmonologist (R.G.). Grading was done according to the American Academy of Sleep Medicine recommendations, that is, AHI score more than or equal to 5 as diagnostic with 5 to 15 as mild, 15 to 30 moderate, and $>30$ severe. $^{15}$

\section{Magnetic Resonance Imaging Pharynx}

Magnetic resonance image acquisition was performed using 1.5T ACHIEVA PHILIPS MRI system (Med Image Systems, Memphis, Tennessee, United States) with use of 16-channel neurovascular coil. Region of interest was from base of skull to laryngeal inlet craniocaudally. Images were obtained in three orthogonal planes: axial, coronal, and sagittal with 4 -mm thick slices. Image acquisition in the axial plane was done from the sphenoid body through the upper larynx, strictly parallel to the hard palate, and the coronal and sagittal planes were perpendicular to the hard palate and parallel to the cervical spine, respectively. Measurements such as diameters of nasopharynx, oropharynx, hypopharynx, and length of tongue and uvula with uvula thickness were made by a single observer (D.K.).

\section{Statistical Analysis}

Data were analyzed using statistical software STATA 14.0 (Texas, United States). Using Kolmogorov-Smirnov test normality of data was checked. Variables following normal distribution were expressed as mean \pm standard deviation, whereas others were expressed as median (minimum and maximum).Comparison of $\mathrm{AHI}$ between treated and nontreated patients were done using MannWhitney $U$ test. Correlation of AHI with cephalometric parameters and serum IGF-1 levels were done using Spearman correlation. A p-value less than 0.05 was considered significant.

\section{Results}

A total of 40 subjects with confirmed acromegaly were screened and after exclusions (one patient did not give consent, three cases fitted in exclusion criteria, and four patients were lost to follow-up), complete data were available on 32 subjects ( 20 men and 12 women) and were taken up for final analysis (-Fig. 1). The mean age of subjects was $42.66 \pm 11.13$ years (range $=26-66$ ) and mean duration before presentation was $7.6 \pm 6.3$ years. Mean BMI of the subjects was $26.4 \pm 4.3 \mathrm{~kg} / \mathrm{m}^{2}$ with mean neck circumference of $35.8 \pm 3.18 \mathrm{~cm}$. The clinical and biochemical parameters are depicted in - Table 1. Headache was the presenting symptom in 56\% and visual field restriction in $22 \%$. Almost $100 \%$ had coarse facial features while $96 \%$ had acral enlargement. A total of 9 out of 32 subjects (28\%) had diabetes mellitus, whereas 13 (41\%) were hypertensive.

Majority of patients, 30 (93.7\%) had pituitary macroadenomas. A total of 20 subjects were treatment naive at the time of assessment and 12 had undergone transsphenoidal surgery (TSS). Only 3 out of 32 (9.3\%) subjects had controlled disease as per the standard acceptable criteria.

OSA as defined by AHI more than 5 was present in $29(90.6 \%)$ patients while none was diagnosed with CSA. The frequency of mild, moderate, and severe OSA was seen in $21.8 \%(n=7), 34.4 \%(n=11)$, and 34.4\% ( $n=11)$, respectively. Among the cephalometric parameters, tongue length correlated strongly with OSA severity $(r=0.56 ; p=0.02)$ while the correlation with uvula length $(r=0.38, p=0.05)$ and uvula thickness ( $r=0.35, p=0.07$ ) was less strong (-Fig. 2).There was no correlation of AHI with body mass index, blood pressure, hemoglobin A1c, serum insulin-like growth factor-1, or human growth hormone levels, or with the form of therapy received ( - Table $\mathbf{2}$ ).

\section{Discussion}

SRBD is often overlooked complication of acromegaly with almost negligible data from Indian subcontinent. The high prevalence of OSA (90\%) in the current study is similar to the previous data from van Haute et $\mathrm{al}^{3}$ and Tsoy et al. ${ }^{4}$ Since most of our patients had longer duration of disease, which was active in the most cases, it could explain such high prevalence. We observed a strong correlation between Apnea-Hypopnea Index (AHI) and tongue length with a 


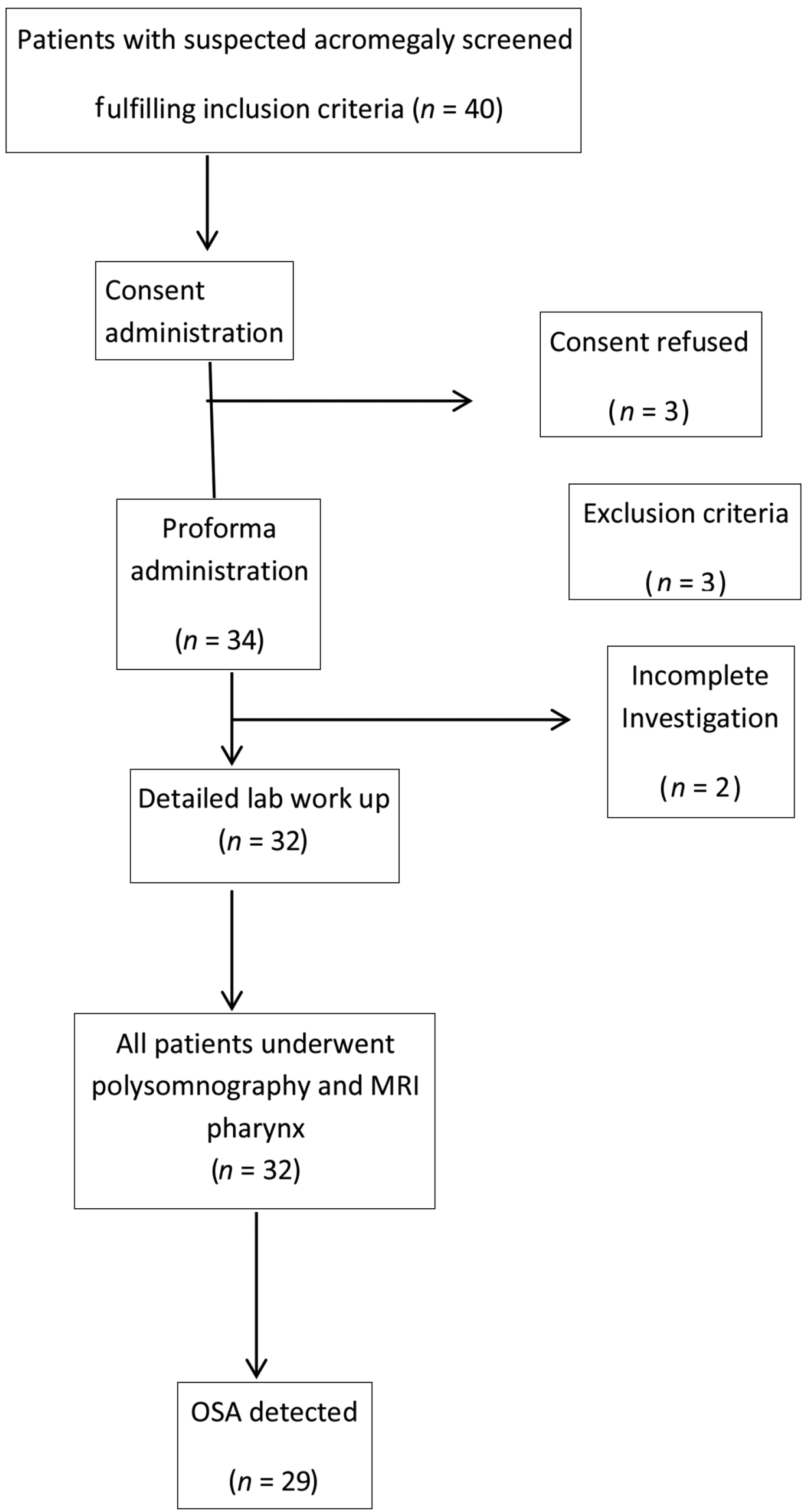

Fig. 1 Pert chart showing flow of subjects in the study. 
Table1 Clinical, biochemical, and hormonal parameters of the study population

\begin{tabular}{|c|c|}
\hline Parameters & Mean \pm SD (range) \\
\hline Age $(y)$ & $42.26 \pm 11.13(26-66)$ \\
\hline Duration of illness $(\mathrm{y})$ & $7.6 \pm 6.3(0.25-32)$ \\
\hline Height (cm) & $165.4 \pm 9.7(142-185)$ \\
\hline Weight $(\mathrm{Kg})$ & $72.5 \pm 13.8(45-100)$ \\
\hline $\operatorname{BMI}\left(\mathrm{Kg} / \mathrm{m}^{2}\right)$ & $26.4 \pm 4.4(21-37.5)$ \\
\hline Waist circumference $(\mathrm{cm})$ & $92.8 \pm 10.1(74-114)$ \\
\hline Hip circumference (cm) & $101.4 \pm 10.6(85-124)$ \\
\hline Neck circumference $(\mathrm{cm})$ & $35.8 \pm 3.18(31-42)$ \\
\hline Systolic BP $(\mathrm{mm} / \mathrm{Hg})$ & $138.6 \pm 14.8(100-160)$ \\
\hline Diastolic BP $(\mathrm{mm} / \mathrm{Hg})$ & $86.8 \pm 10.4(56-110)$ \\
\hline Fasting blood glucose (mg/dL) & $104.3 \pm 23.8(82-241)$ \\
\hline HbA1C (\%) & $5.9 \pm 1.7(4.9-11)$ \\
\hline Serum total cholesterol (mg/dL) & $171.8 \pm 30.2(118-251)$ \\
\hline Serum LDL Cholesterol (mg/dL) & $107.3 \pm 32(84-182)$ \\
\hline Serum HDL Cholesterol (mg/dL) & $40.8 \pm 6.5(30-52)$ \\
\hline Serum triglycerides (mg/dL) & $160.9 \pm 87.7(86-341)$ \\
\hline Serum IGF-1 (ng/mL) & $721.9 \pm 277.6(311-1396)$ \\
\hline Serum fasting hGH (ng/mL) & $22.3 \pm 22.4(0.8-80)$ \\
\hline Serum hGH (1-hour postglucose load) (ng/mL) & $21.3 \pm 21.8(0.6-80)$ \\
\hline Serum hGH (2-hour postglucose load) (ng/mL) & $20.8 \pm 22.8(0.5-80)$ \\
\hline Serum prolactin (ng/mL) & $22.6 \pm 22.4(2.6-104)$ \\
\hline Serum total T4 ( $\mu \mathrm{g} / \mathrm{dL})$ & $7.67 \pm 1.5(5.4-11.6)$ \\
\hline
\end{tabular}

Abbreviations: BMI, body mass index; BP, blood pressure; HbA1C, hemoglobin A1c; HDL, high-density lipoprotein; hGH, human growth hormone; IGF-1, insulin-like growth factor-1; LDL, low-density lipoprotein; SD, standard deviation.

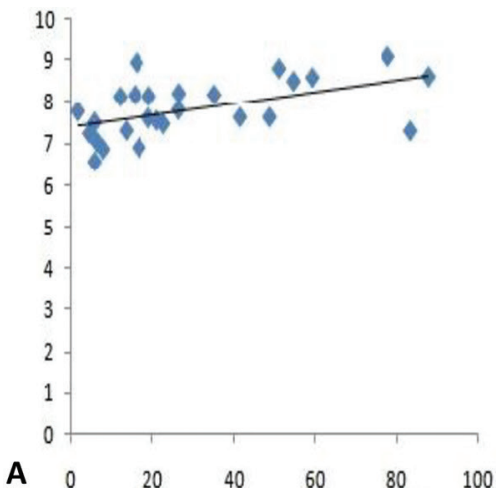

A $\quad 0 \quad 20 \quad 40 \quad 60 \quad 80 \quad 100$
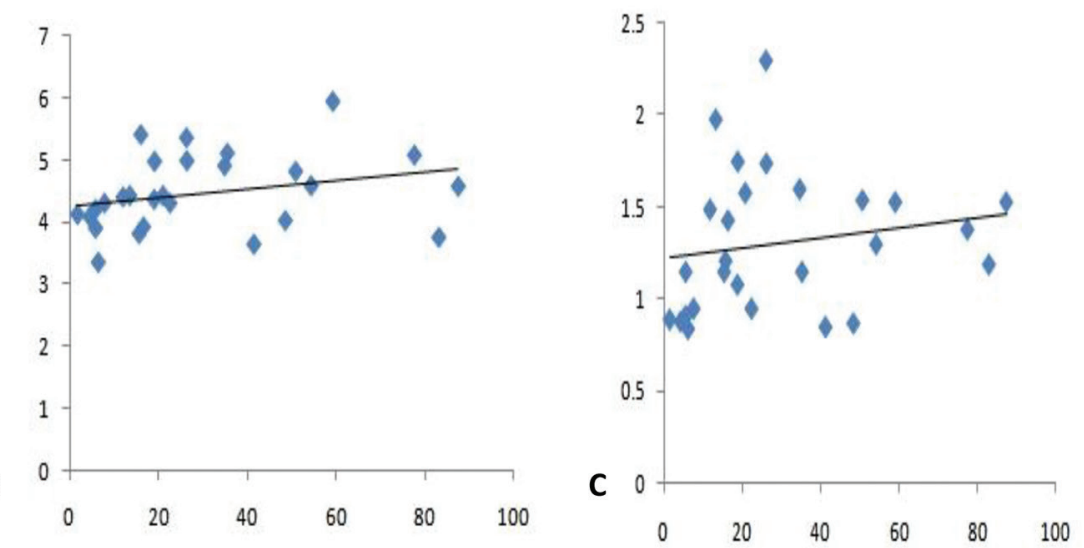

Fig. 2 Correlation of Apnea-Hypopnea Index with (A) tongue length, (B) uvula length, and (C) uvula thickness.

Table 2 Correlation of cephalometric parameters Apnea-Hypopnea Index

\begin{tabular}{|l|l|l|l|}
\hline Parameters & Mean + SD & Spearman correlation coefficient $r$ & $p$-Value \\
\hline Nasopharyngeal diameter $(\mathrm{cm})$ & $7.06 \pm 1.32$ & 0.009 & 0.961 \\
\hline Oropharyngeal diameter $(\mathrm{cm})$ & $1.35 \pm 0.45$ & 0.069 & 0.730 \\
\hline Hypopharyngeal diameter $(\mathrm{cm})$ & $1.19 \pm 0.42$ & 0.121 & 0.550 \\
\hline Tongue length $(\mathrm{cm})$ & $7.79 \pm 0.67$ & 0.569 & 0.002 \\
\hline Uvula length $(\mathrm{cm})$ & $4.5 \pm 0.60$ & 0.385 & 0.050 \\
\hline Uvula thickness & $1.31 \pm 0.37$ & 0.358 & 0.072 \\
\hline
\end{tabular}

Abbreviation: SD, standard deviation. 
weaker correlation to uvular length and thickness suggesting the role of these anatomical abnormalities in the pathophysiology of OSA among acromegaly. These results are supported by the observations by Castellani et al, ${ }^{16}$ who showed higher uvula thickness and tongue length in acromegaly patients with OSA compared with those without OSA. On the contrary, van Haute et $\mathrm{al}^{3}$ and Sesmilo et al ${ }^{17}$ showed no correlation between severity of OSA and cephalometric parameters. The differences could be attributed to variations in image acquisition and interpretation in these small cohorts. Castellani et $\mathrm{al}^{16}$ measured the cephalometric parameters using a software program whereas in the study by van Haute et al, ${ }^{3}$ the measurements were done by a certified radiologist like in our study. The mode of imaging used was also different in the various studies. van Haute et $\mathrm{al}^{3}$ and Castellani et $\mathrm{al}^{16}$ used MRI for image acquisition, while Ip et al and Parolin et $\mathrm{al}^{8,18}$ and Sesmilo et $\mathrm{al}^{17}$ used computed tomography and teleradiography, respectively.

This is the first data on acromegaly and SRBD among Indian subjects although there are several limitations. These include small sample size, lack of comparative group with disease remission, and absence of long-term follow-up.

We conclude that SRBD is generally overlooked comorbidity of rare condition such as acromegaly and it is highly prevalent in subjects with acromegaly. OSA is almost always the type of SRBD in these cases, and its severity correlates positively with tongue and uvula size. Studies involving large cohort of acromegalic patients with robust follow-up design are required to throw more light on the subject.

\section{Conflict of Interest}

None declared.

\section{References}

1 Fernandez A, Karavitaki N, Wass JA. Prevalence of pituitary adenomas: a community-based, cross-sectional study in Banbury (Oxfordshire, UK). Clin Endocrinol (Oxf) 2010;72(3):377-382

2 Sharma AN, Tan M, Amsterdam EA, Singh GD. Acromegalic cardiomyopathy: epidemiology, diagnosis, and management. Clin Cardiol 2018;41(3):419-425

3 van Haute FR, Taboada GF, Corrêa LL, et al. Prevalence of sleep apnea and metabolic abnormalities in patients with acromegaly and analysis of cephalometric parameters by magnetic resonance imaging. Eur J Endocrinol 2008;158(4):459-465
4 Tsoy UA, Sviryaev YV, Korostovtseva LS, et al. [Clinical features of obstructive sleep apnea syndrome in patients with acromegaly]. Ter Arkh 2015;87(4):47-52

5 Vannucci L, Luciani P, Gagliardi E, et al. Assessment of sleep apnea syndrome in treated acromegalic patients and correlation of its severity with clinical and laboratory parameters. J Endocrinol Invest 2013;36(4):237-242

6 Hernández-Gordillo D, Ortega-Gómez MdelR, Galicia-Polo $\mathrm{L}$, et al. Sleep apnea in patients with acromegaly. Frequency, characterization and positive pressure titration. Open Respir Med J 2012;6:28-33

7 Vouzouneraki K, Franklin KA, Forsgren M, et al. Temporal relationship of sleep apnea and acromegaly: a nationwide study. Endocrine 2018;62(2):456-463

8 Parolin M, Dassie F, Alessio L, et al. Obstructive sleep apnea in acromegaly and the effect of treatment: a systematic review and meta-analysis. J Clin Endocrinol Metab 2020;105(3):dgz116

9 Attal P, Chanson P. Screening of acromegaly in adults with obstructive sleep apnea: is it worthwhile? Endocrine 2018;61(1):4-6

10 Dostálová S, Sonka K, Smahel Z, Weiss V, Marek J. Cephalometric assessment of cranial abnormalities in patients with acromegaly. J Craniomaxillofac Surg 2003;31(2):80-87

11 Guo X, Gao L, Zhao Y, et al. Characteristics of the upper respiratory tract in patients with acromegaly and correlations with obstructive sleep apnoea/hypopnea syndrome. Sleep Med 2018;48:27-34

12 Gadelha MR, Kasuki L, Lim DST, Fleseriu M. Systemic complications of acromegaly and the impact of the current treatment landscape: an update. Endocr Rev 2019;40(1):268-332

13 Dutta P, Hajela A, Pathak A, et al. Clinical profile and outcome of patients with acromegaly according to the 2014 consensus guidelines: impact of a multi-disciplinary team. Neurol India 2015;63(3):360-368

14 Lohman T, Roche F, Mantorell R. Anthropometric standardized reference manual. Abridged Edition. Page 1992;42:44

15 Kapur VK, Auckley DH, Chowdhuri S, et al. Clinical practice guideline for diagnostic testing for adult obstructive sleep apnea: an American Academy of Sleep Medicine Clinical Practice Guideline. J Clin Sleep Med 2017;13(3): 479-504

16 Castellani C, Francia G, Dalle Carbonare L, et al. Morphological study of upper airways and long-term follow-up of obstructive sleep apnea syndrome in acromegalic patients. Endocrine 2016;51(2):308-316

17 Sesmilo G, Resmini E, Sambo M, et al; ACROSAHS study group. Prevalence of acromegaly in patients with symptoms of sleep apnea. PLoS One 2017;12(9):e0183539

18 Ip MS, Tan KC, Peh WC, Lam KS. Effect of Sandostatin LAR on sleep apnoea in acromegaly: correlation with computerized tomographic cephalometry and hormonal activity. Clin Endocrinol (Oxf) 2001;55(4):477-483 